\title{
Salud mental en reclusos. Un análisis pre-post intervención psicosocial con grupo control de comparación
}

\author{
Miguel Jesús Bascón Díaz* y Virginia Vargas Girón \\ Departamento de Psicología Experimental. Facultad Psicología. Universidad de Sevilla. (España).
}

\begin{abstract}
Resumen: La literatura científica advierte de la elevada presencia de trastornos mentales en el medio penitenciario. Por ello, en este trabajo nos planteamos evaluar la salud psicosocial y la autorregulación de reclusos en relación a un grupo control de participantes no reclusos; adicionalmente tratamos de conocer la incidencia de la variable tiempo de reclusión y analizar el efecto intragrupo pre-post entrenamiento en habilidades sociales y comunicativas. La muestra estuvo compuesta por 20 varones, 10 reclusos (cinco con más de un año de condena y cinco con menos) y 10 participantes sin antecedentes delictivos, a los que se les administró el cuestionario de salud GHQ-28 y la escala de autorregulación MAPA. Se aplicó la prueba no paramétrica Mann-Whitney $(U)$ para el cálculo de probabilidades y el test de Cramer $(V)$ como indicador del tamaño de efecto. Los resultados indicaron que la reclusión no implicó necesariamente peor salud y autorregulación, que el tiempo de condena no ejerció excesiva influencia sobre estas dos dimensiones y que el taller de habilidades sociales no pareció, según el análisis pre-postest, haber producido efectos sobre la salud y la autorregulación de los reclusos. Finalmente, se discuten algunas reflexiones generales así como nuevas propuestas para mejorar actuaciones futuras.

Palabras clave: salud; autorregulación; reclusos; habilidades sociales; tiempo de reclusión.
\end{abstract}

\section{Introducción}

Este trabajo se centra en el estudio de la salud psicosocial y la autorregulación del comportamiento en reclusos, y su posible relación con aspectos como el tiempo de reclusión y el haber asistido a un curso de formación sobre habilidades sociales y comunicativas. Cuando hablamos de salud psicosocial y autorregulación nos referimos al ajuste que una persona presenta en relación con las demandas que debe afrontar, principalmente en sus relaciones cotidianas. Por tanto remite al equilibrio mental y emocional de los individuos como integrantes de una sociedad, constituyéndose en un elemento fundamental para el desarrollo integral y promoción de hábitos saludables.

De suma importancia son los aspectos relacionados con la salud y la autorregulación entre los reclusos que cumplen condena en las cárceles, teniendo en cuenta que la literatura científica sobre sanidad penitenciaria nos ha venido advirtiendo sobre los efectos negativos de la reclusión y el contexto carcelario (Bermúdez-Fernández, 2006; Blaauw y Kerkhof, 1998; Bustamante et al., 2013; Marín-Basallote y Navarro-Repiso, 2012; Santibañez, 1993). En España en los últimos años se han evidenciado frecuentes cuadros psicopatológicos en el colectivo de reclusos, con una prevalencia que oscila entre el 40\% y el 50\% (Dirección General de Instituciones Penitenciarias del Ministerio del Interior, 2007), y que

* Dirección para correspondencia [Correspondence address]

Miguel Jesús Bascón Díaz. Facultad de Psicología. Dpto. Psicología Experimental. Universidad de Sevilla. C/. Camilo José Cela s/n. 41018. Sevilla (España). E-mail: mjbascon@
Title: Mental health in prisoners. Pre-post analysis of psychosocial intervention with control group of comparison.

Abstract: Scientific literature indicates a high incidence of mental disorders in prisons. Thus, the aim of this study is to evaluate psychosocial health and self-regulation of inmates compared with a control group of non-inmates participants. In addition, we try to determine the incidence of the variable time spent in prison and to analyze the effect of training in social and communicative skills. The sample consisted of 20 males, 10 inmates (five with more than one year of sentence and five with less) and 10 participants with no criminal history. Participants were administered the health questionnaire GHQ-28 and the MAPA scale of self-regulation. Non-parametric Mann-Whitney $(U)$ tests were used to calculate probabilities and Cramer's test $(V)$ as an indicator of effect size. Results evidenced that reclusion was not necessarily associated to poorer health and selfregulation, that the influence of the length of the sentence on these two dimensions was small and that training in social and communicative skills did not produce significant effects on inmates' health and self-regulation. To conclude, some general reflections as well as new proposals to improve future interventions are discussed.

Key words: health; self-regulation; inmates; social skills; time in prison.

llega a ser cinco veces superior a la de la población general (Vicens et al., 2011). Éste fenómeno pudiera estar relacionado con la carencia de herramientas y destrezas psicológicas implicadas en la resolución y afrontamiento de problemas, así como con la puesta en marcha de conductas dominadas por respuestas emocionales y de evitación (Mohino, Kirchner y Forns, 2004).

Otro aspecto importante en relación al grado de disfunción psicológica de los reclusos es el tiempo de reclusión o transcurrido en la cárcel. Kirchner (2003) halló que los reclusos que llevaban más tiempo internados presentaban menor índice psicopatológico, por la posible reducción del estrés que les producía el inicio de la reclusión. Este hecho podría indicar una mayor adaptación a las condiciones de la cárcel de quienes llevaban ya algún tiempo recluidos, en comparación con aquellos otros que tuvieron un ingreso más reciente en dicho escenario.

Así, teniendo en cuenta el menoscabo psicológico que pueden ocasionar la reclusión y el tiempo de condena, pensamos que podría resultar determinante para la salud de los reclusos la puesta en marcha de acciones formativas dirigidas a la dotación de recursos personales y grupales, factores que además contribuyen a su futura reinserción social. En esta línea, Guerreiro, Conboy y Martins (2007) estudiaron en el sur de Portugal a 12 reclusos de sexo masculino participando durante cuatro meses en un programa de intervención y educación social, encontrando mejoras significativas en sus competencias sociales (agresividad, retraimiento, inmadurez y resolución de problemas en las relaciones sociales). Entendemos que la mejora de estas dimensiones sociales del comportamiento puede relacionarse positivamente con la salud y la au- 
torregulación de los reclusos; por ello diseñamos un curso de formación y desarrollo de habilidades sociales y comunicativas dirigido a los internos que se describe en el apartado de método.

Hemos de tener en cuenta que la salud psicosocial ha de entenderse como un concepto multidimensional, compuesto por factores que hacen referencia a la capacidad de adaptación, la madurez intelectual, emocional y conductual, la superación de problemas y frustraciones, la identidad individual y social, o el control de los impulsos (Asociación ProSalud Psicosocial, 2012). Por ello, los problemas de salud psicosocial pueden ser variados, al igual que sus causas, pero todos ellos implican en el individuo cierta pérdida en la capacidad para dirigirse y conducirse dentro de un colectivo o comunidad.

En este sentido, un reconocido enfoque a la hora de analizar el comportamiento dirigido a la búsqueda de la salud es el que se ha venido centrando en la autorregulación (García y Días, 2007). Diversas investigaciones han tratado este tema de estudio. Así, Baumeister y Vohs (2004) consideraban la autorregulación como un esfuerzo del individuo para alterar sus estados interiores o sus comportamientos dirigido al logro de objetivos, intereses o desafíos. En esta misma línea Ridder y De Wit (2006) destacaron la implicación de procesos como la percepción de agentividad, la capacidad de conducción hacia metas, la toma de decisiones, el control de las necesidades y la activación de los estados mentales y emocionales, mientras que Barkley (2004) entendía la autorregulación como la monitorización y adaptación del comportamiento orientado el logro de objetivos.

El trabajo que aquí se presenta apuesta por una aproximación discursiva como propuesta para el análisis de la autorregulación. Partimos del correlato que propuso Billig (1987) entre pensamiento y discurso, considerando las acciones discursivas como herramientas del pensamiento de acuerdo a los presupuestos de Bakhtin (1986), y teniendo en cuenta su destacada funcionalidad sobre la resolución de problemas (Quignard, 2005).

Pero quizás lo más interesante de cara al tema que nos ocupa es la doble dimensión que el discurso presenta. En el plano interpersonal se pone de manifiesto en las situaciones comunicativas, pero el discurso tiene además un efecto en el plano intrapersonal, sobre el propio hablante. Generalmente el habla produce consecuencias sobre los sentimientos, pensamientos o acciones del auditorio pero también sobre quien realiza dicha acción, dada la propensión retórica y narrativa sobre sí mismo y sobre los demás (Duncan, 1989). Así, en nuestro trabajo asumimos que el discurso puede funcionar como un instrumento que articula la autorregulación de la actividad del individuo, constituyéndose un plano reflexivo y metacognitivo que modifica el medio interno del hablante y su experiencia (Belinchón, Rivière e Igoa, 1992). En este sentido, Kuhn, Wang y Li (2010) resaltaron el valor del discurso como herramienta de autoconocimiento pudiéndose relacionar con funciones de regulación del comportamiento, planificación y evaluación.
En definitiva, en nuestro estudio nos planteamos tres objetivos. Primero evaluar la salud psicosocial y la autorregulación de reclusos en relación a un grupo control de participantes no reclusos, antes y después de la realización por parte de los primeros de un curso sobre habilidades sociales y comunicativas. Segundo conocer la incidencia del tiempo de reclusión. Finalmente, pretendemos valorar en los reclusos las posibles diferencias intragrupo en las variables de salud y autorregulación, que permitan conocer el posible efecto del curso en términos de ganancias pre-postest.

Las predicciones apuntan que el grupo de reclusos presentará peor salud psicosocial y autorregulación. También esperamos que los internos con menos tiempo de condena muestren peores puntuaciones en estos marcadores psicológicos. Adicionalmente, hipotetizamos una mejora tras haber cursado el taller de habilidades sociales y comunicativas. Los posibles hallazgos de nuestro trabajo podrían aportar directrices para el diseño de programas de tratamiento dirigidos a mejorar la salud mental y el comportamiento autorregulado de este colectivo.

\section{Método}

\section{Participantes}

La muestra estudiada estuvo compuesta por un total de 20 participantes varones españoles, de los cuales 10 fueron reclusos de un centro penitenciario de Sevilla y otros 10 ciudadanos sin antecedentes personales en materia delictiva, que constituyeron el grupo control de comparación. En este sentido, cabe señalar que dispusimos de una muestra de reclusos que puede considerarse de tamaño reducido debido a la dificultad para reclutar a un mayor número de participantes para el estudio. Ello fue debido a una restringida accesibilidad a algunos servicios de la institución penitenciaria y al incremento que se produjo en las medidas de seguridad y actuación. Todos los participantes reclusos se encontraban internos en un módulo de la Unidad Mixta (cumplimiento y preventivo). Se ofrecieron a participar voluntariamente tras haber sido informados, seleccionándose aquellos que cumplían ciertos criterios de inclusión previamente establecidos: (a) conducta no disruptiva y colaboradora dentro del centro penitenciario; (b) no realización de algún curso de habilidades sociales o participación en otra intervención psicológica; (c) que la condena durara al menos lo mismo que dicho curso (5 meses). No se incluyeron participantes con dificultades de comprensión de las tareas a realizar en el estudio.

La elección de los participantes del grupo control se efectuó de manera no aleatoria, ya que se procuró seleccionar a personas con características sociodemográficas similares (edad, sexo, estudios) a las del grupo experimental, teniéndose también en cuenta que no hubiesen realizado previamente ningún curso de habilidades sociales o recibido alguna intervención psicológica. Igualmente, la participación fue voluntaria tras el preceptivo consentimiento informado. 
Como se observa en la Tabla 1 el total de la muestra tenía un promedio de edad de 38.5 años $(D T=4.882$; rango 3048), un nivel educativo generalmente medio-bajo y ocupaciones diversas que se correspondían con su estrato social. En cuanto al grupo de reclusos, la edad promedio fue de
35.8 años $(D T=4.565$; rango 30-44); cinco tenían un tiempo de condena inferior al año y otros cinco superior. El grupo control presento un promedio de edad de 41.2 años $(D T=$ 3.645; rango 35-48).

Tabla 1. Características sociodemográficas de los participantes.

\begin{tabular}{|c|c|c|c|c|c|}
\hline Participantes & Edad & Sexo & Estudios & Ocupación & T. Condena \\
\hline Experimental 1 & 44 & Varón & Superiores & Guitarrista & -1 año \\
\hline Experimental 2 & 33 & Varón & Primaria & Albañil & -1 año \\
\hline Experimental 3 & 38 & Varón & Secundaria & Mantenimiento & -1 año \\
\hline Experimental 4 & 35 & Varón & Secundaria & Encofrador & -1 año \\
\hline Experimental 5 & 40 & Varón & Primaria & Cocinero & -1 año \\
\hline Experimental 6 & 32 & Varón & Secundaria & Cuida Caballos & +1 año \\
\hline Experimental 7 & 35 & Varón & Secundaria & Comercial & +1 año \\
\hline Experimental 8 & 31 & Varón & Sin Estudios & Albañil & +1 año \\
\hline Experimental 9 & 30 & Varón & Primaria & Nunca trabajó & +1 año \\
\hline Experimental 10 & 40 & Varón & Secundaria & Nunca trabajó & +1 año \\
\hline Control 1 & 40 & Varón & Secundaria & Camionero & --- \\
\hline Control 2 & 35 & Varón & Secundaria & Albañil & --- \\
\hline Control 3 & 39 & Varón & Secundaria & Pintor & --- \\
\hline Control 4 & 38 & Varón & Secundaria & Apicultor & --- \\
\hline Control 5 & 42 & Varón & Secundaria & Marroquinero & --- \\
\hline Control 6 & 43 & Varón & Sin Estudios & Jubilado & --- \\
\hline Control 7 & 41 & Varón & Primaria & Frutero & --- \\
\hline Control 8 & 45 & Varón & Secundaria & Marroquinero & --- \\
\hline Control 9 & 41 & Varón & Secundaria & Herrero & --- \\
\hline Control 10 & 48 & Varón & Primaria & Panadero & --- \\
\hline
\end{tabular}

\section{Instrumentos}

Se aplicaron dos instrumentos: GHQ-28 (General Health Questionnaire) y MAPA (Measure Auto-regulation by Psychosocial Adjustment).

El Cuestionario General de Salud (GHQ) en su versión de 28 ítems (Goldberg, 1978) se divide en cuatro factores: somatización, ansiedad, disfunción social y depresión. Se trata de un instrumento ampliamente utilizado en investigación que ha mostrado su fiabilidad y validez en el análisis de la salud psicosocial. En nuestro caso, obtuvimos una adecuada consistencia interna. En el pretest, para la totalidad del instrumento $(\alpha=.86)$, somatización $(\alpha=.89)$, ansiedad $(\alpha=$ $.89)$, disfunción social $(\alpha=.70)$ y depresión $(\alpha=.96)$. En cuanto al postest, se obtuvo en la totalidad $(\alpha=.89)$, somatización $(\alpha=.92)$, ansiedad $(\alpha=.83)$, disfunción social $(\alpha=$ .85) y depresión $(\alpha=.94)$. Respecto al análisis intragrupo, obtuvimos $(\alpha=.88),(\alpha=.93),(\alpha=.71),(\alpha=.77)$ y $(\alpha=.97)$ para el instrumento, somatización, ansiedad, disfunción social y depresión, respectivamente. Para el análisis estadístico utilizamos su puntuación de investigación (cero, uno, dos o tres para cada opción de los ítems) de forma que un aumento de puntuación en el GHQ significaba peor salud.

MAPA es un instrumento diseñado para la ocasión y construido en base al sistema de tres categorías de respuestas para el análisis de la autorregulación propuesto por Autor (2014). Contiene 12 supuestos hipotéticos repartidos equitativamente en tres escenarios o subescalas: situaciones de la vida cotidiana, pareja y familia. La elección de estos tres ám- bitos responde a nuestro interés por conocer si las respuestas diferían entre contextos. Para su realización se pidió a los participantes que respondieran aquello que harían ante cada supuesto. Las respuestas fueron codificadas y clasificadas en base al citado sistema de categorías (se describe en diseño y análisis) en relación con la medida de autorregulación: autorregulación impositiva, autorregulación acomodadiza y autorregulación resolutiva. Estas tres modalidades de respuesta fueron puntuadas como uno, dos y tres, respectivamente, representando un gradiente de menor a mayor nivel de autorregulación.

En relación a MAPA hemos de decir que existe una versión adaptada para adolescentes, aunque el instrumento original está diseñado e indicado para adultos. Ninguno de ellos ha sido administrado en otras culturas ni idiomas distintos al español. Para su confiabilidad se elaboró una batería con varios ítems representativos por cada una de las situaciones, para posteriormente llevar a cabo un proceso de depuración entre dos expertos hasta la delimitación de las 12 que constituyeron el test. Durante el proceso de revisión se procuraron controlar posibles sesgos evitando ítems tendenciosos, largos (más de 20 palabras), oraciones ambiguas, dobles negaciones y expresiones polarizadas. Para su selección se tuvieron en cuenta los puntajes de cada uno y su correlación con el puntaje de su subescala, eliminándose o revisándose aquellos con correlaciones inferiores a .30.

En cuanto a la validez, los ítems fueron puestos a prueba en una muestra de 72 sujetos. En concreto, en el presente estudio el análisis de fiabilidad mostró una adecuada consisten- 
cia interna. En el pretest, la totalidad del instrumento $(\alpha=$ .94), la subescala situaciones de la vida cotidiana $(\alpha=.77)$, pareja $(\alpha=.92)$ y familia $(\alpha=.82)$. En el postest, el instrumento $(\alpha=.94)$, situaciones de la vida cotidiana $(\alpha=.71)$, pareja $(\alpha=.95)$ y familia $(\alpha=.80)$. En el análisis intragrupo se obtuvo $(\alpha=.71)$ en el instrumento, $(\alpha=.73)$ en la situaciones de la vida cotidiana, $(\alpha=.70)$ en pareja y $(\alpha=.69)$ en familia. Finalmente, se elaboró una guía abreviada con orientaciones e instrucciones de cara a su administración; en ella se incluyeron algunas especificaciones sobre criterios estadísticos de puntuación y baremación así como pautas para la interpretación de las medidas.

\section{Procedimiento}

Tras el correspondiente consentimiento informado de todos los participantes, el estudio se dividió en cuatro fases.

Fase 1. Aplicación de GHQ-28 y MAPA a los 10 reclusos antes del curso.

Se procedió a administrar estos dos instrumentos antes de comenzar el curso de habilidades sociales y comunicativas con la finalidad de obtener una medida de salud y de autorregulación previa al mismo (pretest).

Fase 2. Realización del curso de habilidades sociales y comunicativas.

Se llevó a cabo la impartición del taller únicamente a los 10 reclusos, que tuvo como finalidad la adquisición de competencias para el afrontamiento de situaciones interpersonales adversas, de cara a la mejora de la convivencia en el centro penitenciario y la futura reinserción social y laboral. Durante cinco meses se realizaron un total de 20 sesiones semanales con una duración aproximada de 90 minutos cada una; fueron impartidas por dos psicólogas en un aula del módulo de respeto del propio recinto carcelario. Se desarrollaron dinámicas de presentación y conocimiento grupal, escucha activa, estilos de comunicación, autoestima, aprender a debatir, identificar problemas y soluciones, expresar emociones y relajación.

Fase 3. Aplicación de GHQ-28 y MAPA a los 10 internos tras el curso.

Finalizado el curso se volvieron a administrar los dos instrumentos al grupo de reclusos con objeto de obtener una nueva medida de salud y autorregulación que permitiera conocer su evolución (postest).

Fase 4. Aplicación de GHQ-28 y MAPA a los 10 participantes no reclusos (grupo control).

Se efectuó con ellos una única aplicación con objeto de comparar su estado de salud y autorregulación en relación con aquellos participantes encarcelados.

\section{Diseño y análisis}

De acuerdo con lo expuesto por Ato, López y Benavente (2013) se planteó un diseño cuasiexperimental con grupo control no equivalente (DGCNE). Debido a la falta de alea- torización en la selección muestral se analizaron todas las variables que pudieran presentar una concomitancia diferencial significativa entre grupos, de cara a hacerlos lo más comparables posible (Shadish, Cook y Campbell, 2002); se encontró equivalencia en todas ellas. Así, se logró la complementariedad entre grupos controlando ciertas características sociodemográficas de los participantes como edad $\left(\chi^{2}=12.66, p=\right.$ .474 ), sexo (todos varones) y estudios $\left(\chi^{2}=1.53, p=.675\right.$ ), así como el hecho no haber realizado ningún curso de habilidades sociales previamente. Asimismo, de cara a que los resultados no se viesen distorsionados procedimos a realizar un emparejamiento de tipo individual para restringir la selección de controles, caso a caso en función de las características o condiciones referidas anteriormente.

Manejamos dos variables explicativas como fueron el tipo de situación, con dos condiciones: estar recluido (grupo experimental de reclusos) vs. no estarlo (grupo control de comparación), y el tiempo transcurrido en la cárcel por los reclusos: menos de un año vs. más de un año. Como variables de respuesta se analizaron la salud y la autorregulación con las que se efectuaron dos mediciones, una antes y otra después de la impartición del curso sobre habilidades sociales y comunicativas a modo de análisis pretest-postest.

La salud fue medida en base a los cuatro factores del GHQ-28 mientras que la autorregulación se estudió mediante el sistema de categorías articulado en MAPA. Las mismas fueron obtenidas mediante un proceso de saturación y adaptación de la propuesta de Rahim y Bonoma (1979) y utilizada por Monteiro, Serrano y Rodríguez (2012), que cruzando el interés propio con el interés por los demás clasifica las respuestas en base a tres categorías: autorregulación impositiva, autorregulación acomodadiza y autorregulación resolutiva. En el ejemplo se ilustran las tres respuestas en relación al Supuesto $\mathrm{n}^{\circ}$ 6: Con ilusión ahorras y regalas un viaje a tu pareja, pero ella te dice que prefería otra cosa.

Autorregulación impositiva (AI). Mandatos y dominaciones que satisfacen lo propio y no lo ajeno representando el menor grado de autorregulación. Ejemplo de respuesta: Si no va me voy con otra porque me ha costado mucho trabajo pagarlo.

Autorregulación acomodadiza (AA). Sumisión y evitación que anteponen lo ajeno sobre lo propio; suelen dar lugar a una moderada autorregulación orientada por y hacia los demás. Ejemplo de respuesta: No baría nada, si no le gusta no iríamos.

Autorregulación resolutiva (AR). Integración de posturas y búsqueda de acuerdo que suponen el mayor grado de autorregulación. Ejemplo de respuesta: Intentaría hacer otro que nos guste a los dos.

Como método de fiabilidad del sistema de categorías se llevó a cabo una triangulación entre investigadores a partir de los registros individuales (Cohen y Crabtree, 2008). Se codificaron el 30\% de los enunciados en dos sesiones paralelas y las discrepancias se resolvieron mediante un tercer juez. Tras obtener una óptima concordancia dada por un coeficiente $\mathrm{K}$ $=.88$ el sistema de categorías se aplicó al 100\% de los enunciados. El análisis fue realizado con ayuda del software informático ATLAS.TI 7 y SPSS 22. 
El tratamiento estadístico de los datos se efectuó mediante la aplicación de la prueba no paramétrica de MannWhitney. La prueba de Mann-Whitney supone una alternativa sólida a la $t$ de Student, que no requiere parámetros de distribución específicos y es apropiada para muestras pequeñas con grupos desiguales (McKnight y Najab, 2010). Se hallaron las medias grupales $(M)$ y sus desviaciones típicas $(D T)$, los valores del estadístico $(U)$, sus probabilidades asumiendo un nivel de confianza del $95 \%(p<.05)$ y el test de Cramer $(V)$ como indicador del tamaño de efecto. Mostramos los efectos de las variables explicativas tipo de situación (reclusión/no reclusión) y tiempo de reclusión (menos de un año/más de un año) sobre los indicadores de salud (GHQ28) y autorregulación (MAPA), atendiendo a los dos momentos de medida (pretest y postest). Posteriormente, se presentan los análisis intragrupo (pre-post intervención) en dichos indicadores.

\section{Resultados}

\section{Pretest. Aplicación de GHQ-28 y MAPA antes del curso formativo}

Respecto a los indicadores de salud medidos a través del GHQ-28, como indica la Tabla 2, se obtuvo solo un valor significativo en función de la variable reclusión/no reclusión $(U=10.00, p=.012, V=.658)$, con tamaño de efecto grande. Concretamente los participantes no reclusos presentaron una puntuación media más alta, es decir, peor funcionamiento social, que aquellos otros que estaban recluidos $(M=1.04$ vs. $M=0.71$. En cuanto a la somatización, ansiedad y depresión no se encontraron diferencias relevantes por el hecho de estar recluido en relación a los que gozaban de libertad.

Referente a la media de autorregulación con MAPA, como se observa en la Tabla 2, se halló influencia de la variable reclusión/no reclusión sobre las tres subescalas, con tamaños de efecto grandes: situaciones de la vida cotidiana $(U=7.00$, $p=.006, V=.717)$, pareja $(U=15.50, p=.044, V=.738) \mathrm{y}$ familia $(U=12.00, p=.023, V=.797)$. Así, quienes estaban recluidos presentaron un mayor valor medio de autorregulación en las tres subescalas, respecto a los que no lo estaban: situaciones de la vida cotidiana $(M=2.67$ vs. $M=1.82)$, pareja $(M=2.85$ vs. $M=2.10)$ y familia $(M=2.67$ vs. $M=$ 1.95).

Centrándonos en el grupo de reclusos representado en la Tabla 3, tan solo se apreció un efecto significativo de la variable tiempo de reclusión sobre la salud, concrétamente sobre el factor depresión y con tamaño de efecto muy intenso $(U=0.00, p=.019, V=1)$. En este sentido, se observó un mayor valor medio de marcadores depresivos en los reclusos con menos de un año de condena que en aquellos otros con más de un año $(M=1.52 v s$. $M=0)$. Los otros tres parámetros de salud medidos con el GHQ no se vieron condicionados.

Tabla 2. Pretest: Tipo de Situación, GHQ-28, MAPA.

\begin{tabular}{lccccc}
\hline & $\begin{array}{c}\text { Reclusión } \\
M(D T)\end{array}$ & $\begin{array}{c}\text { No Reclusión } \\
M(D T)\end{array}$ & $U$ & $p$ & $V$ \\
\hline Somatización & $0.51(0.70)$ & $0.52(0.48)$ & 30.00 & .620 & \\
Ansiedad & $0.97(0.49)$ & $0.72(0.67)$ & 23.50 & .258 & \\
Disf. Social & $0.71(0.21)$ & $1.04(0.22)$ & 10.00 & .012 & .658 \\
Depresión & $0.65(1.01)$ & $0.11(0.21)$ & 27.50 & .391 & \\
Situación VC & $2.67(0.27)$ & $1.82(0.62)$ & 7.00 & .006 & .717 \\
Pareja & $2.85(0.24)$ & $2.10(0.84)$ & 15.50 & .044 & .738 \\
Familia & $2.67(0.31)$ & $1.95(0.69)$ & 12.00 & .023 & .797 \\
\hline Nota: $M=$ Media; $D T=$ Desviación Típica.
\end{tabular}

Respecto a la autorregulación medida con MAPA también se encontró (véase Tabla 3) un único efecto significativo del tiempo de internamiento, de magnitud muy grande, sobre las situaciones de la vida cotidiana $(U=0.50, p=.046, V=$ .842). Así, quienes llevaban menos tiempo recluidos presentaron mayor valor medio en la capacidad de autorregulación sobre este tipo de situaciones, en relación a quienes llevaban más $(M=2.91$ vs. $M=2.50)$.

Tabla 3. Pretest: Tiempo de Reclusión, GHQ-28, MAPA.

\begin{tabular}{|c|c|c|c|c|}
\hline & $\begin{array}{c}\text { Reclusión }<1 \text { año } \\
M(D T)\end{array}$ & $\begin{array}{c}\text { Reclusión }>1 \text { año } \\
M(D T)\end{array}$ & $U \quad p$ & $V$ \\
\hline Somatización & $1.00(0.89)$ & $0.14(0.20)$ & 1.00 .074 & \\
\hline Ansiedad & $0.90(0.21)$ & $1.03(0.67)$ & $6.00 \quad 1$ & \\
\hline Disf. Social & $0.57(0.14)$ & $0.82(0.21)$ & 2.00 .146 & \\
\hline Depresión & $1.52(1.01)$ & $0(0)$ & 0.00 .019 & 1 \\
\hline Situación VC & $2.91(0.14)$ & $2.50(0.20)$ & 0.50 .046 & 842 \\
\hline Pareja & $2.83(0.28)$ & $2.87(0.25)$ & 5.50 .823 & \\
\hline Familia & $2.66(0.28)$ & $2.68(0.37)$ & 6.00 & \\
\hline
\end{tabular}

Nota. $M=$ Media; $D T=$ Desviación Típica.

\section{Postest. Aplicación de GHQ-28 y MAPA tras el cur-} so formativo

En relación con la salud, el análisis de los cuatro factores del GHQ-28 mostró (véase Tabla 4) solo el efecto significativo de la variable reclusión/no reclusión sobre el funcionamiento social, aunque con un tamaño de efecto muy grande $(U=12.00, p=.023, V=1)$. De este modo, los participantes no reclusos seguían teniendo una puntuación media más alta que los reclusos, lo que indicó una mayor disfunción en este factor $(M=1.04 v s . M=0.69)$.

En cuanto a la autorregulación se observó (véase Tabla 4) influencia significativa del hecho de estar o no recluido sobre las tres subescalas, con tamaños de efecto grandes: situaciones de la vida cotidiana $(U=8.00, p=.007, V=.694)$, pareja $(U=12.50, p=.017, V=.627)$ y familia $(U=11.00, p=$ $.017, V=.802)$. En relación a ello, los internos mostraron mayor autorregulación media en todas las subescalas: situaciones de la vida cotidiana $(M=2.60 v s$. $M=1.82)$, pareja $(M$ $=2.96$ vs. $M=2.10)$ y familia $(M=2.67 v$ s. $M=1.95)$. 
Tabla 4. Postest: Tipo de Situación, GHQ-28, MAPA.

\begin{tabular}{lccccc}
\hline & $\begin{array}{c}\text { Reclusión } \\
M(D T)\end{array}$ & $\begin{array}{c}\text { No Reclusión } \\
M(D T)\end{array}$ & $U$ & $p$ & $V$ \\
\hline Somatización & $0.91(0.84)$ & $0.52(0.48)$ & 24.50 & .301 & \\
Ansiedad & $1.02(0.40)$ & $0.72(0.67)$ & 22.50 & .219 & \\
Disf. Social & $0.69(0.61)$ & $1.04(0.22)$ & 12.00 & .023 & 1 \\
Depresión & $0.44(0.68)$ & $0.11(0.21)$ & 18.00 & .424 & \\
Situación VC & $2.60(0.19)$ & $1.82(0.62)$ & 8.00 & .007 & .694 \\
Pareja & $2.96(0.09)$ & $2.10(0.84)$ & 12.50 & .017 & .627 \\
Familia & $2.67(0.23)$ & $1.95(0.69)$ & 11.00 & .017 & .802 \\
\hline Nota: $M=$ Media; $D T=$ Desviación Típica. & & & &
\end{tabular}

$\mathrm{Al}$ analizar las cuatro dimensiones de salud del GHQ en función del tiempo de reclusión se apreció un único efecto significativo (véase Tabla 5), concretamente sobre el factor depresión y con una magnitud muy grande $(U=0.00, p=$ $.019, V=1)$. Así, aquellos participantes recluidos menos de un año presentaron más sintomatología depresiva $(M=1.04$ vs. $M=0$ ). En el resto de factores (somatización, ansiedad y función social) no se hallaron diferencias significativas.

Por lo que respecta a la medida de autorregulación obtenida mediante MAPA no se encontró ningún efecto significativo del tiempo de reclusión (véase Tabla 5), es decir, esta variable no se relacionó con ninguna de las tres dimensiones estudiadas: situaciones de la vida diaria, pareja y familia.

Tabla 5. Postest: Tiempo de Reclusión, GHQ-28, MAPA.

\begin{tabular}{lccccc}
\hline & $\begin{array}{c}\text { Reclusión }<1 \\
\text { año } \\
M(D T)\end{array}$ & $\begin{array}{c}\text { Reclusión }>1 \\
\text { año } \\
M(D T)\end{array}$ & $U$ & $p$ & $V$ \\
\hline Somatización & $0.95(0.65)$ & $0.89(1.06)$ & 5.00 & .721 & \\
Ansiedad & $0.85(0.14)$ & $1.14(0.52)$ & 4.50 & .593 & \\
Disf. Social & $1(0.86)$ & $0.46(0.29)$ & 3.00 & .271 & \\
Depresión & $1.04(0.67)$ & $0(0)$ & 0.00 & .019 & 1 \\
Situación VC & $2.75(0.05)$ & $2.50(0.20)$ & 1.50 & .076 & \\
Pareja & $2.91(0.14)$ & $3(0.03)$ & 4.00 & .248 & \\
Familia & $2.66(0.28)$ & $2.68(0.23)$ & 5.50 & .844 & \\
\hline Nota. & & & & & \\
& & &
\end{tabular}

Nota. $M=$ Media; $D T=$ Desviación Típica.

\section{Pretest-Postest intragrupo}

Finalmente, el análisis de las posibles diferencias intragrupo (pre-post intervención) sobre la salud (GHQ) y la autorregulación (MAPA) indicó que ni el grupo de reclusos con menos de un año de internamiento ni los que llevaban más experimentaron variaciones estadísticamente significativas entre las fases pretest y postest. Atendiendo a la comparación de medias, en la Tabla 6 observamos como todos los valores estadísticos indicaron la ausencia de cambios relevantes entre una y otra fase en cada una de las dimensiones estudiadas: somatización, ansiedad, disfunción social, depresión, situaciones de la vida cotidiana, pareja y familia.
Tabla 6. Pretest-Postest Intragrupo: Tiempo de Reclusión, GHQ-28, MAPA.

\begin{tabular}{|c|c|c|c|c|c|}
\hline & $\begin{array}{l}\text { Pretest } \\
M(D T)\end{array}$ & $\begin{array}{l}\text { Postest } \\
M(D T)\end{array}$ & $U$ & $p$ & $V$ \\
\hline \multicolumn{6}{|c|}{ Reclusión $<1$ año } \\
\hline Somatización & $1(0.89)$ & $0.95(0.65)$ & 4.00 & .825 & \\
\hline Ansiedad & $0.90(0.21)$ & $0.85(0.14)$ & 4.00 & .822 & \\
\hline Disf. Social & $0.57(0.14)$ & $1(0.86)$ & 4.00 & .822 & \\
\hline Depresión & $1.52(1.01)$ & $1.04(0.67)$ & 2.00 & .275 & \\
\hline Situación VC & $2.91(0.14)$ & $2.75(0.05)$ & 1.50 & .114 & \\
\hline Pareja & $2.83(0.28)$ & $2.91(0.14)$ & 4.00 & .796 & \\
\hline Familia & $2.66(0.28)$ & $2.66(0.28)$ & 4.50 & 1 & \\
\hline \multicolumn{6}{|c|}{ Reclusión $>1$ año } \\
\hline Somatización & $0.14(0.20)$ & $0.89(1.06)$ & 3.50 & .180 & \\
\hline Ansiedad & $1.03(0.67)$ & $1.14(0.52)$ & 7.00 & .767 & \\
\hline Disf. Social & $0.82(0.21)$ & $0.46(0.29)$ & 2.00 & .080 & \\
\hline Depresión & $0(0)$ & $0(0)$ & 8.00 & 1 & \\
\hline Situación VC & $2.50(0.20)$ & $2.50(0.20)$ & 8.00 & 1 & \\
\hline Pareja & $2.87(0.25)$ & $3(0.03)$ & 6.00 & .317 & \\
\hline Familia & $2.68(0.37)$ & $2.68(0.23)$ & 8.00 & 1 & \\
\hline
\end{tabular}

Nota: $M=$ Media; $D T=$ Desviación Típica.

\section{Discusión y conclusiones}

Procedemos a organizar y sistematizar la discusión en secciones de acuerdo a los tres objetivos planteados.

\section{Relación entre tipo de situación, estado de salud y autorregulación}

Se apreció una escasa influencia de la variable reclusión/no reclusión sobre la salud, es decir, los reclusos únicamente se diferenciaron de los no reclusos en un solo factor del GHQ (disfunción social); así, y en contra de lo esperado, quienes estaban recluidos presentaron mejor funcionamiento social que aquellos que gozaban de libertad. La misma circunstancia se produjo tras la realización del curso de habilidades sociales y comunicativas.

En el caso de la autorregulación todas las subescalas que conforman el instrumento MAPA mostraron variaciones en función de la situación de los participantes. En relación a ello, tanto las situaciones de la vida cotidiana como la pareja y la familia representaron ámbitos de relación en los que los reclusos obtuvieron mejores puntuaciones que los no reclusos, yendo el hallazgo de nuevo en contra de lo hipotetizado. Las diferencias también se mantuvieron similares antes y después de la realización del curso.

Estos resultados no validan nuestras hipótesis iniciales cuyas predicciones apuntaban a peores puntuaciones en reclusos. A pesar de la tendencia a publicar los hallazgos positivos (Dickersin, 1997), creemos que nuestros datos pueden resultar provechosos para el campo de la sanidad penitenciaria. Así, el grupo de reclusos mostró mejor salud y sobre todo mejor capacidad de autorregulación que aquellos otros participantes que gozaban de libertad. Pensamos que los mejores índices en autorregulación pudieran estar relacionados con el propio carácter normativo, de control y cumplimiento explícito de medidas que supone el contexto carcelario. Es 
probable que la estructuración de funciones junto con la organización de las actividades dentro del centro penitenciario haya podido incidir en que los reclusos estén más reglados y regulados. Por tanto podría ser interesante en un futuro indagar en el estudio de las diferencias entre internos y condenados en régimen abierto para tratar de establecer posibles efectos correctores asociados al encarcelamiento.

\section{Relación entre tiempo de reclusión, estado de salud y autorregulación}

Respecto a la incidencia del tiempo de reclusión solo se observaron diferencias en un único factor de salud del GHQ pero con tamaño de efecto muy intenso. Más concretamente, quienes llevaban recluidos menos de un año arrojaron mayores indicadores de depresión, repitiéndose antes y después del curso de habilidades sociales y comunicativas. En esta ocasión los resultados fueron en consonancia con lo esperado en nuestro trabajo, coincidiendo con los obtenidos por Kirchner (2003) quien observó menor psicopatología en los reclusos que llevaban más tiempo internados. Este hecho pudiera estar condicionado por la inoculación del estrés inicial, la adaptación al contexto y a la apropiación de una subcultura carcelaria que les hace compartir, conocer las circunstancias del medio, adoptar un rol cooperador y sentirse un miembro más de la comunidad penitenciaria (BermúdezFernández, 2006; García y Pacheco, 2012). En otras palabras, pensamos que de acuerdo a la idea de Ordóñez (citado en García y Pacheco, 2012) se podría producir una posible reconstrucción identitaria en los reclusos institucionalizados, que les permite desarrollar nuevos instrumentos y estrategias de regulación psicológica a través de diferentes etapas o fases. Consideramos que este fenómeno asociado al estudio de la identidad también puede resultar de interés para futuros trabajos.

En cuanto a la medida de autorregulación podemos apreciar que tan solo existió variación en la subescala relativa a las situaciones de la vida cotidiana. Ello se tradujo en que los reclusos que llevaban cumpliendo condena menos de un año se ajustaron mejor a este tipo de escenarios que aquellos que llevaban más tiempo, algo no esperado en nuestra hipótesis. En esta ocasión las diferencias desaparecieron tras la realización del curso.

\section{Diferencias intragrupo (pre-post intervención)}

Por último analizamos la evolución de la salud psicosocial y la autorregulación en el grupo de reclusos dividiéndolo en dos subgrupos en función del tiempo de reclusión, con el propósito de calibrar el efecto del curso formativo en habilidades sociales y comunicativas. En contra de lo esperado, ni los internos con menos de un año de cumplimiento de condena ni los que llevaban más experimentaron variaciones significativas entre la fase pretest y postest. Por tanto no se registraron cambios significativos atribuibles directamente al curso en ninguna de las dimensiones de salud del GHQ (so- matización, ansiedad, disfunción social y depresión), ni en las tres subescalas de autorregulación de MAPA (situaciones de la vida cotidiana, pareja y familia).

En relación a la falta de efectividad de la intervención nos surgen algunas reflexiones.

En primer lugar pudiera ser debida a las características que presentaban los reclusos. Tal como mencionamos en el método se seleccionaron aquellos que presentaron mayor colaboración y menos comportamiento disruptivo, por lo que posiblemente ya contaran con ciertas habilidades sociales y comunicativas básicas; recordemos que antes de la realización del curso (pretest) ya mostraron adecuados marcadores de salud y autorregulación. Teniendo en cuenta lo anterior se hace preciso realizar una selección adecuada y ajustada de los internos participantes a las características de la acción formativa.

Relacionado con lo anterior, en el ámbito aplicado debemos considerar rigurosamente el efecto de nuestras intervenciones en la organización, valores y significados de la comunidad donde trabajemos. Así, es posible que nuestro curso constituyera una actividad ciertamente aislada, de insuficiente duración y poco coordinada con la política general de tratamiento del centro. Por ello sería recomendable la sincronía entre los diferentes agentes que intervienen en la sanidad penitenciaria (Murillo y Ruiz, 2004).

Finalmente, de acuerdo con Del Rey (2004) creemos que existen dos elementos determinantes que hay que procurar para el éxito de las intervenciones en el medio carcelario. Por un lado la creación de un ambiente adecuado, tanto físico como psicosocial. Por otro lado el entrenamiento y la implicación de todo el personal que trabaja con los internos de manera que su actividad trascienda a la mera custodia. Ninguno de estos dos aspectos fueron especialmente controlados ni tenidos en cuenta en nuestro trabajo.

En definitiva, resumiendo los principales hallazgos de este estudio podemos obtener varias conclusiones. Primero que el hecho de estar recluido no implicó necesariamente peor salud y autorregulación, más bien en nuestro estudio sucedió lo contrario. Segundo que los reclusos mostraron mejor autorregulación que los participantes no reclusos. Tercero que el tiempo de reclusión no tuvo excesiva influencia sobre las dos dimensiones estudiadas. Cuarto que el curso no produjo efectos destacables sobre la salud y la autorregulación en los reclusos según indica el análisis intragrupo pretest-postest.

Entre las limitaciones que presenta este trabajo podemos encontrar el reducido tamaño muestral dado, como se he apuntado en el apartado de participantes, por la dificultad de acceso y restricciones del contexto carcelario. Aunque encontramos trabajos con un número similar de participantes (Castro, López-Castedo y Sueiro, 2009; Guerreiro et al., 2007) creemos que la potencia de los datos se vería optimizada si eleváramos dicho número, a la vez que ello permitiría emplear pruebas paramétricas de contraste bajo supuestos de normalidad y homocedasticidad. También sería recomendable una revisión, depuración y mejora de la escala MAPA, así 
como un estudio longitudinal que permitiera el seguimiento de los participantes. Paralelamente podríamos explorar las cuestiones tratadas en una muestra de mujeres reclusas y tratar de testar en futuros trabajos las posibles diferencias o semejanzas por razón de género.

Otras mejoras teórico-metodológicas con vocación aplicada podrían dirigirse a correlacionar los cuatro indicadores de salud con las tres subescalas de autorregulación, para esclarecer si una mayor autorregulación va asociada a una mejor salud y/o viceversa; en este sentido, García y Días (2007) consideraban la autorregulación como un medio para lograr

\section{Referencias}

Ato, M., López, J. J. y Benavente, A. (2013). Un sistema de clasificación de los diseños de investigación en psicología [A classification system for research designs in psychology]. Anales de Psicología, 29 (3), 1038-1059. http://dx.doi.org/10.6018/analesps.29.3.178511.

Asociación Pro-Salud Psicosocial. (2012). ¿Qué es la Salud Psicosocial? [What is the Psychosocial Health?]. Recuperado de http://www.pro-saludpsicosocial.org/inicio.html

Autor (2014). Adolescencia, género y conflicto. El discurso argumentativo como herramienta de autorregulación [Adolescence, gender and conflict. Argumentative discourse as a tool for self-regulation]. Boletín de Psicología, 110, 7-19. Recuperado de http://www.uv.es/seoane/boletin/boletin.html

Bakhtin, M. (1986). Speech genres and other late essays. Austin: University of Texas Press.

Barkley, R. A. (2004). Attention-deficit/hyperactivity disorder and selfregulation. En R. F. Baumeister y K. D. Vohs (Eds.), Handbook of selfregulation: Research, theory, and applications (pp. 301-323). New York: Guilford.

Baumeister, R. F. y Vohs, K. D. (2004). Handbook of Self-Regulation: Research, Theory, and Applications. New York: Guilford.

Belinchón, M., Rivière, A. e Igoa, J. M. (1992). Psicología del lenguaje. Investigación y teoría [Psychology of language. Research and theory]. Madrid: Trotta.

Bermúdez-Fernández, J. I. (2006). Efectos psicológicos del encarcelamiento [Psychological effects of imprisonment]. En J. C. Sierra, E. M. Jiménez y G. Buela-Casal (Eds.), Psicología forense: manual de técnicas y aplicaciones (pp. 348-371). Madrid: Biblioteca Nueva.

Billig, M. (1987). Arguing and thinking, a rhetorical approach to social psychology. Cambridge, MA: Cambridge University Press.

Blaauw, E. y Kerkhof, A. (1998). Suicides in Police Custody in the Netherlands. En R.J. Kosky., H.S., Eshkevari, R. D., Goldney y R. Hassan (Eds.), Suicide Prevention. The Global Context (pp. 131-137). New York: Plenum Press.

Bustamante, R., Paredes-Carbonell, J.J., Aviñó, D., González, J., Pitarch, C., Martínez, L. y Arroyo-Cobo, J. M. (2013). Diseño participativo de una Guía para la Promoción de la Salud Mental en el medio penitenciario [Participatory design of a Guide for Promotion of Mental Health in prisons]. Revista Española de Sanidad Penitenciaria, 15 (2), 44-53. Recuperado de http://www.sanipe.es

Castro, M. E., López-Castedo, A. y Sueiro, E. (2009). Sintomatología asociada a agresores sexuales en prisión [Symptomatology associated with sex offenders in prison]. Anales de Psicología, 25 (1), 44-51. Recuperado de http://revistas.um.es/analesps/index

Cohen, D. J. y Crabtree, B. F. (2008). Evaluative criteria for qualitative research in health care: controversies and recommendations. Annals of Family Medicine, 6 (4), 331-339. doi:10.1370/afm.818.

Del Rey, J. E. (2004). Intervención ambiental con drogodelincuentes encarcelados: Principios, datos y líneas de investigación [Environmental intervention with imprisoned drug addict: Principles, data and research lines]. Revista de Estudios Penitenciarios, 250, 73-96. Recuperado de http://www.interior.gob.es/web/archivos-y-

documentacion/documentacion-y-publicaciones/publicaciones- la salud, fenómeno al que Morgan, Davies y Ziglio (2010) catalogaron como activos para la salud. Recordemos que el conjunto de nuestros hallazgos apuntan a una presencia conjunta de mejores indicios de salud y autorregulación, aunque este es un tema aún nos queda por dilucidar en futuros trabajos.

Agradecimientos.- Este trabajo se ha podido llevar a cabo gracias a la colaboración de todas las personas participantes en el mismo, especialmente las del centro penitenciario.

descargables/publicaciones-periodicas-anuarios-y-revistas-/revista-deestudios-penitenciarios

Dickersin K. (1997). How important is publication bias? A synthesis of available data. AIDS Education \& Prevention, 9 (A), 15-21. Recuperado de http://www.guilford.com/journals/AIDS-Education-andPrevention/Francisco-Sy/08999546

Dirección General de Instituciones Penitenciarias del Ministerio del Interior. (2007). Estudio sobre la salud mental en el medio penitenciario [Study] about mental bealth in prisons]. Recuperado de http://www.msc.es/organizacion/sns/planCalidadSNS/ boletinAgencia/boletin10/estudio_saludMental_medio_penitenciario.pdf.pdf

Duncan, H. (1989). Communication and social order. New Brunswick: Transaction Publishers.

García, J. A. y Días, P. (2007). Análisis relacional entre los factores de protección, resiliencia, autorregulación y consumo de drogas [Relational analysis between protective factors, resilience, self-regulation and illegal substance use]. Salud y drogas, 7 (2), 309-332. Recuperado de http:/ / www.haaj.org/index.php?journal=haaj\&page=index

García, N. y Pacheco, M. (2012). La construcción subjetiva de las consecuencias del encarcelamiento: punto de vista del recluso [Subjective construction of imprisonment consequences: Prisoner's viewpoint]. Acción Psicológica, $9 \quad$ (2), 21-33. doi: http://dx.doi.org/10.5944/ap.9.2.4101

Goldberg D. (1978). Manual of the General Health Questionnaire. Windsor: NFER Publishing.

Guerreiro, H., Conboy, J. y Martins, A. (2007). Desenvolvimento de competências sociais e redução da dependência em reclusos [Development of social skills and reducing reliance on prisoners]. Psicologia, Teoria, Investigação e Prática, 12 (1), 55-72.

Kirchner, T. (2003). Estrategias de afrontamiento y nivel de psicopatología en jóvenes presidiarios. Relación con el tiempo de reclusión y situación penitenciaria [Coping strategies and psychopathology in young male inmates: relationship with time spent in prison and remand]. Acción Psicológica, 2 (3), 199-21. http://dx.doi.org/10.5944/ap.2.3.531

Kuhn, D., Wang, Y. y Li, H. (2010). Why Argue? Developing Understanding of the Purposes and Values of Argumentive Discourse. Discourse Processes, 48 (1), 26-49. doi: 10.1080/01638531003653344

McKnight, P. E. y Najab, J. (2010). Mann-Whitney U Test. Corsini Encyclopedia of Psychology, 1. doi: 10.1002/9780470479216.corpsy0524

Marín-Basallote, N. y Navarro-Repiso, C. (2012). Estudio de la prevalencia de trastorno mental grave (TMG) en los centros penitenciarios de Puerto I, II y III del Puerto de Santa María (Cádiz): nuevas estrategias en la asistencia psiquiátrica en las prisiones [Study of the prevalence of severe mental disorders in the penitentiaries Puerto I, II y III of Puerto de Santa Maria (Cadiz): new strategies of psychiatric care in prisons]. Revista Española de Sanidad Penitenciaria 14 (3), 80-85. http://dx.doi.org/10.4321/S1575-06202012000300002

Mohino, S., Kirchner, T. y Forns, M. (2004). Coping Strategies in Young Male. Prisoners. Journal of Youth and Adolescence, 33 (1), 41-50. doi: 10.1023/A:1027382229951

Monteiro, A. P., Serrano, G. y Rodríguez, D. (2012). Estilos de gestión del conflicto, factores de personalidad y eficacia en la negociación [Conflict 
management styles, personality factors and effectiveness in the negotiation]. Revista de Psicología Social, 27 (1), 97-109. doi: 10.1174/021347412798844042

Morgan A., Davies M. y Ziglio E. (2010). Health Assets in a Global Context: Theory Methods Action. New York: Springer.

Murillo, A. y Ruiz, A. B. (2004). Un programa de tratamiento destinado al interno y su actitud frente al delito [A treatment program for prisoner and attitude to crime]. Revista de Estudios Penitenciarios, 250, 97-131. Recuperado de http://www.interior.gob.es/web/archivos-ydocumentacion/documentacion-y-publicaciones/publicaciones-

descargables/publicaciones-periodicas-anuarios-y-revistas-/revista-deestudios-penitenciarios

Quignard, M. (2005). A collaborative model in dyadic problem-solving interactions. En F. H, Van Eemeren y P, Houtlosser, (Eds), Argumentation in practice (pp. 69-86). Philadelphia: John Benjamins Publishing Company.
Rahim, M. y Bonoma, T. (1979). Managing organizational conflict: A model for diagnosis and intervention. Psychological Reports, 44 (3c), 1323-1344. doi: $10.2466 /$ pr0.1979.44.3c.1323

Ridder, D. y De Wit, J. (2006). Self-Regulation in Health Behavior. Chichester: Wiley.

Santibáñez, R. (1993). ¿Reformar la ley o reformar la realidad? Personalidad y tiempo de estancia en prisión [Reform law or reality? Personality and length of stay in prison]. Eguzkilore, 7, 147-156.

Shadish, W. R., Cook, T. D. y Campbell, D. T. (2002). Experimental and Quasi-experimental designs for generalized causal inference. New York, NY: Houghton Mifflin. Recuperado de https://depts.washington.edu/methods/readings/Shadish.pdf

Vicens, E., Tort, V., Dueñas, R.M., Muro, A., Pérez, F., Arroyo, J.M., ... Sarda, P. (2011). The prevalence of mental disorders in Spanish prisons. Journal Criminal Behaviour and Mental Health, 21 (5), 321-32. doi: 10.1002/ cbm. 815 .

(Artículo recibido: 14-01-2015; revisado: 02-03-2015; aceptado: 02-06-2015) 